\title{
Observed Orbital Eccentricities
}

\author{
Helmut A. Abt ${ }^{1}$ \\ ${ }^{1}$ Kitt Peak National Observatory, Box 26732, Tucson, AZ 85726-6732, USA \\ email: abt@noao.edu
}

\begin{abstract}
The eccentricities of catalogued binaries show that tidal interactions extend to periods of at least 1000 days. The maximum periods for complete circularization probably depend on age, rather than mass. For the longest periods all eccentricities are equally probable. For shorter periods the highest eccentricities disappear first. For the same periods, the SB2's have larger mean eccentricities than the SB1's, in accord with Keplers Third Law.
\end{abstract}

Keywords. binaries: spectroscopic, binaries: visual, stellar: evolution

This is a discussion of the eccentricities in main-sequence spectroscopic and visual binaries with known orbital elements. I collected data on 553 SBs from Dimitri Pourbaix's superb on-line compilation and 616 VBs from the U.S. Naval Observatory's excellent online Sixth Catalog. Orbital elements of quality 1 were not included.

If one plots the eccentricities as a function of period, one first sees the well-known fact that systems with periods less than a few days have been circularized by tidal interaction. The theory for that has been developed by Zahn $(1975,1977)$ and Tassoul \& Tassoul (1992). But for longer periods the data show a seemingly random distribution. If we fit a mathematical curve to those points, we get a growth curve that goes from zero eccentricity for the shorter periods and then asymptotically approaches 0.5 for the longer periods. But it makes more sense to compute mean eccentricities for bins in the periods because then we can derive error estimates. Figure 1 shows a sample graph, in this case for $221 \mathrm{G}$ dwarfs.

The curves for stars of other spectral types are similar but with various starting points and asymptotic values. Table 1 shows a summary from the fitted curves.

The maximum period for complete circularization seems to vary from about 1.5 days for early-type stars to 4.3 days for GK stars. But is that period a function of primary mass or age? Mathieu \& Mazeh (1988) and Mathieu et al. (1990) derived numbers like 10-11 days for G stars in old clusters, Latham et al. (1988) gave values up to 18 days for halo stars. Mathieu \& Mazeh (1988) proposed that the maximum circularization period is a function of age. Therefore I suggest that the periods shown in the table are mostly dependent on age, not mass. This agrees with Zahns calculations.

We see that the asymptotic eccentricity for very long periods averages $0.52 \pm 0.02$ for all types. If we look at the mean eccentricities for 111 F0-M5 dwarfs with orbital periods greater than $10^{5}$ days $(275 \mathrm{yr})$, we see that all eccentricities are equally probable. That means that when wide binaries are formed, all eccentricities have the same probability of occurring.

When we look at the frequencies of various eccentricities for shorter periods, the higher eccentricities disappear, i.e., for $32<P<100$ days, there are no binaries with eccentricities greater than 0.8 . For $10<P<32$ days, there are no eccentricities greater than 0.7 . For $3.2<P<10$ days, there are no eccentricities greater than 0.3 . 


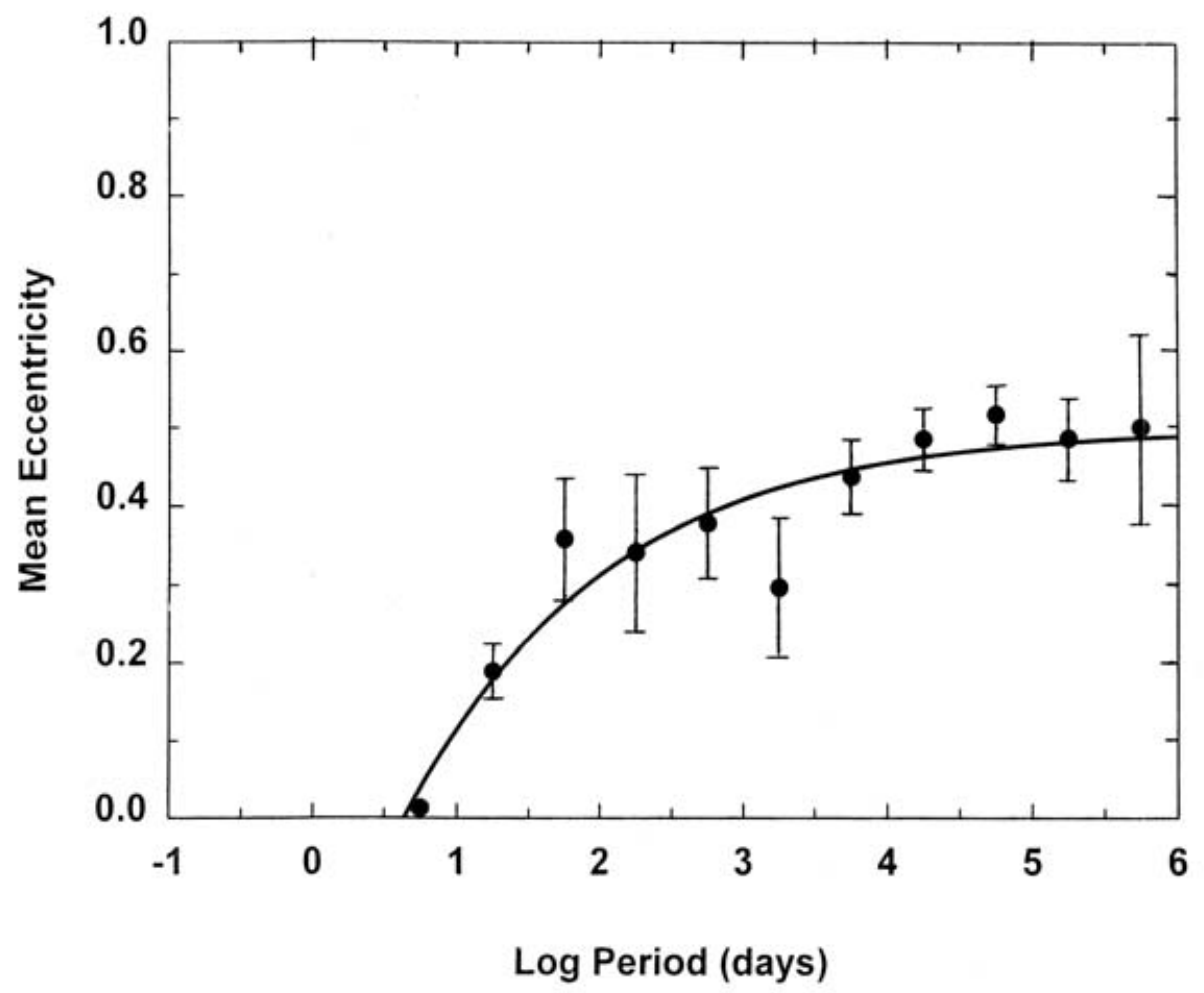

Figure 1. The mean eccentricities of 221 binaries with G dwarf primaries are plotted against orbital periods. The fitted mathematical curve shows a maximum period for total circularization of 4.33 days and an asymptotic eccentricity of 0.50 .

Table 1. Results from fitted curves for all spectral types

\begin{tabular}{lccc}
$\begin{array}{l}\text { Spectral } \\
\text { Range }\end{array}$ & $\begin{array}{c}\text { Number of } \\
\text { Binaries }\end{array}$ & $\begin{array}{c}\text { Max. Circularization } \\
\text { Period (days) }\end{array}$ & $\begin{array}{c}\text { Asymptotic } \\
\text { Eccentricity }\end{array}$ \\
\hline & & & \\
B0-B9.5 & 131 & 1.41 & $0.55 \pm 0.02$ \\
A0-A5 & 148 & 1.60 & $0.53 \pm 0.09$ \\
A6-F0 & 85 & 2.24 & $0.55 \pm 0.07$ \\
F0-F9 & 363 & 1.98 & $0.58 \pm 0.06$ \\
G0-G9 & 221 & 4.33 & $0.50 \pm 0.06$ \\
K0-M5 & 136 & 4.27 & $0.44 \pm 0.06$
\end{tabular}

We would expect that the tidal interactions should be stronger for binaries having more massive secondaries. We can assume that SB2's have more massive secondaries than SB1's. Note that for binaries with periods up to about 1000 days the tidal effects are substantial because their mean eccentricities have been reduced. We can look at a sample of 143 SB2's and 118 SB1's of primary types F0-M5. For periods between 10 and 1000 days where tidal effects partially reduce the eccentricities, the mean eccentricities for the SB2's are consistently higher. Consider Keplers Third Law. For a fixed period, the separation of the components is larger for SB2's than for SB1's, so the tidal interaction is less. That explains why the eccentricities remain larger for SB2's. 
We conclude that:

(a) tidal effects in binaries extend to periods of about 1000 days,

(b) the maximum period for complete circulations probably depends mostly on age, rather than primary mass,

(c) for the longest periods all eccentricities are equally probable,

(d) for shorter periods the highest eccentricities disappear first, and

(e) for binaries of the same periods, the SB2's have smaller tidal effects than the SB1's.

These results for early-type stars were published in $A p J 629,507,2005$ and for the latetype stars in the November 10, 2006 ApJ (ApJ 651, 1151, 2006).

\section{References}

Latham, D.W., Mazeh, T., Carney, B. W., McCrosky, R.E., Stefanik, R.P., \& Davis, R.J. 1988, AJ 96,567

Mathieu, R.D. Latham, D.W., \& Griffin, R.F. 1990, AJ 100, 1859

Mathieu, R.D. \& Mazeh, T. 1988, ApJ 326, 256

Tassoul, J.-L. \& Tassoul, M. 1992, ApJ 395, 259

Zahn, J.-P. 1975, A\&BA 41, 329

Zahn, J.-P. 1977, A\&̈A 57, 383

\section{Discussion}

JEAN-Louis Halbwachs: I am wondering about the large $<e>$ that you found for SB2. With the Geneva group (Halbwachs et al. 2005) we compared the eccentricities of G-K MS binaries with $q \sim 1$ to that of the others, and we found that, for a given $P$, twins have a smaller median $e$ than the other binaries. Did you also consider $q$ ?

ABT: I collected values of $q$ for the SB2's, but did not use them. But since the difference in $\langle e\rangle$ between SB2's and SB1's is only about $2 \sigma$, I hesitated to subdivide the SB2's. But I will look into the difference between Halbwachs et al. (2005) and my data. Thank you. 